\title{
Optimal Combined Heat and Power System Scheduling in Smart Grid
}

\author{
Kan Zhou*, Jianping Pan ${ }^{+}$, and Lin Cai* \\ ${ }^{*}$ Dept. of Electrical \& Computer Engineering; ${ }^{+}$Dept. of Computer Science \\ University of Victoria, Victoria, BC, Canada
}

\begin{abstract}
Combined Heat and Power (CHP) systems are well known for their high efficiency and relatively low emissions. Existing CHP economic dispatch schemes do not use the energy buffer to minimize the average cost in the long term. Motivated by the queueing analysis and buffer management solutions in data communication systems, in this paper, we investigate how to use a battery pack and a water tank to optimize the average cost for the CHP systems by jointly considering the real-time electricity price, renewable energy generation, energy buffer states, etc. We first formulate the queueing models for the CHP systems, and then propose an algorithm based on the Lyapunov optimization technique which does not need any statistical information about the system dynamics. The optimal control actions are obtained by solving a non-convex optimization problem. We then discuss when it can be converted into a convex optimization problem. Since the battery pack queue and water tank queue are correlated by the CHP, the capacity relationship between them is further explored. Through the theoretical performance analysis, we also show the tradeoff between the cost saving and the energy buffer capacity. Finally, the effectiveness of the proposed algorithms is evaluated with practical data.
\end{abstract}

Index Terms - Combined Heat and Power, Lyapunov Optimization, Smart Grid

\section{INTRODUCTION}

Combined heat and power (CHP) systems can generate both electricity and thermal energy simultaneously from a single fuel source, and can achieve a much higher energy efficiency than generating electricity and heat separately [1]. The use of CHP systems can also reduce greenhouse gas emissions. As a result, CHP systems are becoming increasingly popular. Existing works try to minimize the total fuel cost by optimizing the operation points of CHP systems in each time slot. They are called combined heat and power economic dispatch (CHPED) problems [2], [3]. However, in the conventional CHPED problem, there is no energy buffer which can be used to store excessive energy when the energy price is low to save it for later use. With large batteries, such as distributed PHEVs, flywheel energy storage, electrochemical battery, super-conducting magnetic energy storage, etc. [4], [5], and water tanks which can be used to store heat in the form of hot water, it is possible to optimize the total energy cost in the long term. Motivated by the queueing analysis and buffer management solutions in data communication systems, in this paper, we investigate how to use a battery pack and a water tank to optimize the average cost for the CHP systems. We first formulate the queueing models for the CHP systems. Then, we use the Lyapunov optimization technique to minimize the average energy cost by dispatching the electricity and natural gas in each time slot. Both the stochastic electricity and hot water demands from users are met in each time slot. Since the CHP systems generate electricity and heat simultaneously, the energy queues of the battery pack and the water tank are related. Therefore we cannot use the stochastic network optimization framework to solve the problem directly. We consider two different types of CHP systems. The first one is a conventional CHP which uses natural gas as the fuel and has a fixed electricity-heat generation ratio, while the second one uses renewable energy and has an adjustable electricityheat generation ratio.

The contributions of this paper are three-fold. First, we propose a comprehensive model from the perspective of a commercial customer, which incorporates both the electricity and thermal energy queues. We investigate the relationship of these two queues to minimize the average cost. Second, we propose an algorithm to approximately achieve the optimal average cost, considering the limited capacities of the battery pack and the water tank. The algorithm does not require any statistical information of the system dynamics such as electricity and hot water demands, etc. To obtain the optimal scheduling decision, we discuss when we can use the specific features of the problem to turn a non-convex optimization problem into a convex one which can be solved in real time. Third, we analyze the performance of our algorithm and validate its effectiveness through extensive simulations.

The rest of the paper is organized as follows. Section II discusses the existing CHPED problems and the application of Lyapunov optimization in smart grid. A general description of the system architecture is given in Section III. Then we discuss the design details of the proposed algorithm in Section IV and analyze its performance. In Section V we discuss how to apply the proposed algorithm to the CHP using renewable energy. Performance evaluation is given in Section VI, followed by concluding remarks and future research issues in Section VII.

\section{RELATED WORK}

Thanks to the ubiquitous communications technologies, it is possible to optimize the provisioning and delivery of various energy sources to achieve a higher efficiency [6], [7]. To provide both electricity and heat economically, the design and operation strategies of CHP systems have been well investigated. [8] discussed operating strategies, such as heat and electricity load following, for three micro-CHP technologies. [9] evaluated four typical operation modes in a hotel based on measured electric and heating loads. [10] analyzed the utilization of micro-CHP systems in conjunction with domestic household appliances. [11] analyzed the cost 
for different fuel-cell systems. These works tried to find the most cost-effective strategies from a system view, and do not consider the detailed control policies. The CHPED problem, first raised in [2], aimed to find the optimal operation point of CHP with minimum energy cost such that both electricity and heat demands were met. A two-level strategy to separate the objective function and constraints was adopted in [2]. Besides the traditional mathematical approaches, evolutionary computation techniques were used to improve the performance [12]. However, in the CHPED problems, optimization was performed to minimize the cost in each time slot. No energy buffer was used to minimize the long-term cost. In addition, it did not consider the stochastic nature of energy demand.

Various optimization technologies have been used to optimize the cost of a smart grid system with energy buffers. T. Chang et al. used the dynamic programming and decomposition approach to minimize the total cost from the perspective of each user and the whole micro-grid, respectively [13]. In their work, the distributions of all the stochastic variables such as the load and real-time price information were assumed to be available from historical data. [14] proposed a threshold based energy storage control policy that minimizes the long-term average grid operational cost based on dynamic programming. Model predictive control (MPC) has also been applied to obtain the optimal control policies. For example, T. G. Hovgaard et al. proposed an economic MPC algorithm to minimize the total cost of distributed power generation plants by using large cold rooms as the energy buffer. Different from these approaches, our proposed algorithm tries to minimize the long-term time average cost without the need to estimate the statistical system dynamics from historical data.

There are also several works which use the Lyapunov optimization technique to construct low complexity energy storage management policies. M. J. Neely et al. minimized the time average cost from the perspective of one user, and guaranteed the worst-case delay for each elastic load in [16]. In [17], the authors used uninterruptible power systems (UPS) in the data center to reduce the electricity bill in a real-time price environment. Their model did not consider renewable energy sources. Guo et al. investigated how to use a household battery to minimize the average electricity cost, considering both inelastic and elastic load in [18]. L. Huang et al. extended their model by considering selling power back to the grid in [19]. Instead of guaranteeing the worst-case delay, [20] guaranteed that the percentage of the delayed elastic load was less than a threshold. These works discussed above only considered one energy buffer, however, the system model discussed in our work includes two energy buffers, the battery pack and the water tank, which are correlated by the CHP system. With two dependent queues, the system model is more complicated and we need to solve a non-convex optimization problem to obtain the optimal control policy. In addition, we illustrate the relationship between the capacity of these two energy buffers and the minimum required capacity to achieve the optimal performance. This paper focuses on the problems closely related to the unique features of the CHP systems.

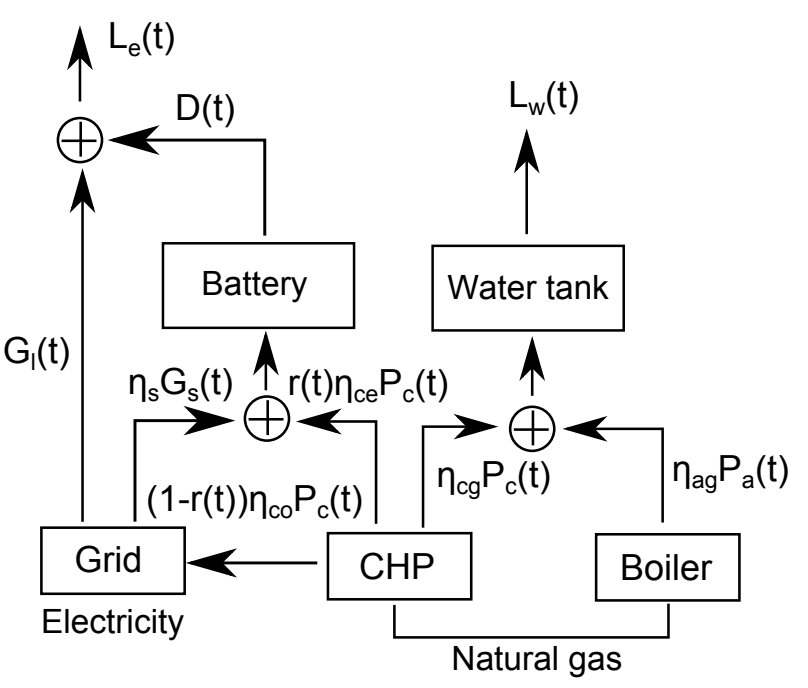

Fig. 1. The CHP system uses natural gas.

Some well-studied applications of Lyapunov optimization in smart grid, such as elastic load queue, worst-case delay, etc, are not discussed here due to the space limitation.

\section{System ModeL}

We consider how to minimize the average energy cost using the CHP device and energy buffers. The mathematical models of this system are discussed in this section.

\section{A. System Architecture}

Fig. 1 gives an overview of the CHP system, such as the one used in a hotel. $L_{e}(t)$ and $L_{w}(t)$ represent the electricity and hot water demands from users in each time slot, respectively, which are stochastic. $L_{e}(t)$ can be met by the electricity discharged from the battery $D(t)$ or bought from the power grid $G_{l}(t) . L_{w}(t)$ is met by the hot water stored in the water tank.

In each time slot, the CHP device can generate electricity, in the amount of $\eta_{c e} P_{c}(t)$, to charge the battery and hot water, in the amount of $\eta_{c g} P_{c}(t)$, to fill the water tank, where $P_{c}(t)$ is the amount of the natural gas consumed by the CHP, $\eta_{c e}$ is the conversion efficiency from natural gas to the amount of the electricity charged to the battery, and $\eta_{c g}$ is the conversion efficiency from natural gas to the amount of hot water. Meanwhile, if the battery is full or the grid electricity price is high, the electricity generated from the CHP, in the amount of $\eta_{c o} P_{c}(t)$, can be sold back to the grid with the conversion efficiency $\eta_{c o}$. The parameter $r(t)$, ranging from 0 to 1 is used to make a tradeoff between the amount of electricity used to charge the battery and that sold to the grid.

Note that we did not let the power generated from the CHP supply the user's electricity demand $L_{e}(t)$ directly in the above model to simplify the analysis. The reason is that we assume the electricity price bought from and sold to the power grid are the same, so whether the electricity is used to supply the user's demand directly or sold back to the grid does not affect 
the total energy cost (if we do not sell the electricity, less electricity is bought from the grid).

Since the electricity price in the real-time electricity market changes according to the supply and demand, in this paper we assume the real-time electricity price $C_{e}(t)$ for the next time slot is known ahead of time. $C_{e}(t)$ is bounded in the range $\left[C_{e, \min }, C_{e, \max }\right]$. On the other hand, the price of the natural gas does not change frequently and the percentage of the change is usually not large, so we assume it is constant in each time slot. However, the proposed algorithm is still applicable if we also consider the real-time gas price because the control decisions of the proposed algorithm are made upon the current system states in each time slot, including the natural gas price.

To minimize the average energy cost in the long term, in each time slot the controller determines the amount of electricity $G_{l}(t)$ and $G_{s}(t)$ bought from the grid to supply the electricity demand and charge the battery, the amount of the natural gas $P_{c}(t)$ consumed by the CHP and the amount of the natural gas $P_{a}(t)$ consumed by the boiler. It also needs to determine the value of $r(t)$ which specifies the dispatch of the generated electricity from the CHP to the battery. The parameter $\eta_{s}$ in Fig. 1 represents the battery charging efficiency, and $\eta_{a g}$ represents the conversion efficiency from natural gas to the amount of hot water using the boiler.

The intuition is that the controller discharges the battery and makes the CHP generate more electricity to meet the high electricity demand or sell to the grid to earn profit when the electricity price is high. On the contrary, the controller charges the battery using the electricity from the grid when the electricity price is low. This problem is challenging because we do not know the distribution of the electricity and hot water demand, nor do we know the distribution of the real-time electricity price. Of course, we can use dynamic programming to estimate the distribution of these stochastic variables, but it is usually computationally complex and may have the "curse of dimensionality" problem [27].

\section{B. Electricity Queueing Model}

In practice, although the lifetime of the battery may be influenced by the charging and discharging process, etc, we do not take them into account. Besides, we assume that the state of charge (SOC) of the battery, viewed as the energy queue of the battery, is linear to simplify our analysis. However, the proposed algorithm will not be largely affected if we incorporate more complicated battery models because the proposed algorithm only needs to know the current battery status to make control decisions.

The SOC level of the battery $B(t)$ evolves according to the following equation:

$$
B(t+1)=B(t)-D(t)+\eta_{s} G_{s}(t)+r(t) \eta_{c e} P_{c}(t) .
$$

Obviously, in any slot $t$, the battery needs to have the following capacity and charge/discharge constraints.

$$
\begin{aligned}
& 0 \leq B(t) \leq B_{\text {max }}, \\
& 0 \leq D(t) \leq D_{\text {max }},
\end{aligned}
$$

$$
0 \leq \eta_{s} G_{s}(t)+r(t) \eta_{c e} P_{c}(t) \leq C_{c h a r}
$$

where $B_{\max }$ is the capacity of the battery, $D_{\max }$ is the maximum discharge rate of the battery, and $C_{c h a r}$ is the maximum charge rate of the battery.

The amount of electricity drawn from the grid in one time slot is also bounded by $P_{e, \max }$ :

$$
\begin{gathered}
0 \leq G_{l}(t)+G_{s}(t) \leq P_{e, \max }, \\
0 \leq G_{l}(t) \leq G_{l, \max }, 0 \leq G_{s}(t) \leq G_{s, \max },
\end{gathered}
$$

where $G_{l, \max }$ and $G_{s, \max }$ are the upper bound of $G_{l}(t)$ and $G_{s}(t)$, respectively. Since the grid can meet the commercial power demand most of the time, we assume $P_{e, \max } \geq L_{e, \max }$ where $L_{e, \max }$ is the upper bound of $L_{e}(t)$.

\section{Water Queueing Model}

The water tank discussed here is assumed an ideal one, so we do not consider heat leakage. A more practical water tank model can easily be applied as we can consider the amount of the heat needed to reheat the water tank as the additional heat demand in the form of hot water from the users.

The amount of hot water stored in the water tank, which is the queue length of the water tank, evolves according to the following equation:

$$
W(t+1)=W(t)-L_{w}(t)+\eta_{c g} P_{c}(t)+\eta_{a g} P_{a}(t),
$$

where $W(t)$ is the water level in the water tank in slot $t$.

Since the amount of the water stored in the water tank should always be bounded by the size of the water tank, we have: $0 \leq W(t) \leq W_{\max }$, where $W_{\max }$ is the capacity of the water tank. In addition, since we assume the hot water demand in each time slot will not exceed $L_{w \text {,max }}$, to make sure that users' demand can always be met even in the worstcase situation, i.e., the hot water demand is always $L_{w, \max }$, we assume the following constraint holds:

$$
L_{w, \max } \leq \eta_{a g} P_{a, \max },
$$

where $P_{a, \max }$ is the maximum amount of the natural gas used by the boiler in each time slot, and $L_{w, \max }$ is the upper bound of the hot water demand in each time slot.

\section{Control Objective}

In each time slot, the total energy cost for the CHP system is the sum of the electricity and natural gas cost minus the amount of the electricity sold to the grid:

$f(t)=C_{e}(t)\left\{G_{l}(t)+G_{s}(t)-(1-r(t)) \eta_{c o} P_{c}\right\}+C_{g}\left\{P_{c}+P_{a}\right\}$,

where $C_{g}$ is the natural gas price.

The control objective is to find a control policy determining the amount of the electricity and natural gas dispatched in each time slot, so as to minimize the long-term average energy cost.

$$
f_{\text {avg }}=\lim _{T \rightarrow \infty} \frac{1}{T} \sum_{i=0}^{T-1} \mathbb{E}\{f(i)\} .
$$




\section{The CHP System Scheduling Algorithm}

In this section, we assume the electricity and hot water demands in each time slot $L_{e}(t), L_{w}(t)$ are independent. The proposed algorithm in this section will solve the following problems. First, given the current states of the CHP system, including the electricity and hot water demand, battery and water tank storage level, electricity price in the current time slot, etc, how to obtain the optimal control decisions with a low computational complexity and can adapt to the stochastic system dynamics while still provide a good performance? Second, what is the minimum capacity of the battery pack and water tank we should have to achieve a given performance requirement? Third, since the CHP can generate both electricity and heat, the battery pack and water tank queues specified in (1) and (7) are dependent. What is the relationship between the capacity of the battery pack and that of the water tank?

According to the system architecture and control objective described in Section III, the problem can be formulated as the following stochastic optimization problem.

Problem One (P-I)

$$
\min _{D(t), r(t), G_{l}(t), G_{s}(t), P_{c}(t), P_{a}(t)} P_{1}=\lim _{T \rightarrow \infty} \frac{1}{T} \sum_{t=0}^{T-1} \mathbb{E}\{f(t)\},
$$

subject to

$$
\begin{gathered}
B(t+1)=B(t)-D(t)+\eta_{s} G_{s}(t)+r(t) \eta_{c e} P_{c}(t), \\
W(t+1)=W(t)-L_{w}(t)+\eta_{c g} P_{c}(t)+\eta_{a g} P_{a}(t), \\
0 \leq B(t) \leq B_{\max } \\
0 \leq W(t) \leq W_{\max } \\
L_{e}(t)=G_{l}(t)+D(t) \\
0 \leq \eta_{s} G_{s}(t)+r(t) \eta_{c e} P_{c}(t) \leq C_{c h a r} \\
0 \leq r(t) \leq 1, P_{c}(t), G_{l}(t), G_{s}(t) \geq 0 \\
0 \leq D(t) \leq D_{\max }
\end{gathered}
$$

The above problem cannot fit into the stochastic optimization framework directly mainly because of the battery and water tank capacity constraints (14) and (15). Specifically, stochastic optimization can only guarantee the average energy generation equals the average consumption in the long term, but cannot provide a hard bound on the difference between the generation and consumption in any time slot. To solve this problem, we take the expectation on the two sides of (12) and (13), which leads to the following relaxed problem:

\section{Problem Two (P-II)}

$$
\min _{D(t), r(t), G_{l}(t), G_{s}(t), P_{c}(t), P_{a}(t)} P_{1}=\lim _{T \rightarrow \infty} \frac{1}{T} \sum_{t=0}^{T-1} \mathbb{E}\{f(t)\},
$$

subject to

$$
\begin{gathered}
\overline{D(t)}=\eta_{s} \overline{G_{s}(t)}+\eta_{c e} \overline{r(t) P_{c}(t)}, \\
\overline{L_{w}(t)}=\eta_{c g} \overline{P_{c}(t)}+\eta_{a g} \overline{P_{a}(t)},
\end{gathered}
$$

and (16), (17), (18), (19).

P-II fits the stochastic optimization framework, so we can solve it using existing algorithms [21], [22]. Obviously, only when the solutions to P-II can meet the constraints (14) and (15) for $\forall t \in T$, they are also feasible to P-I. To reach this objective, we define two constants $\theta$ and $\varepsilon$. The intuition is that by adjusting these two constants appropriately, we can make the solutions to P-II also be feasible to P-I.

To start, we define two queues $E(t)$ and $X(t)$ :

$$
\begin{gathered}
E(t)=B(t)-\theta, \\
X(t)=W(t)-\varepsilon .
\end{gathered}
$$

The constants $\theta$ and $\varepsilon$ are two queue offsets, which are used to guarantee that the two queues $B(t)$ and $W(t)$ are bounded.

From (12) and (13), we can obtain the queueing dynamics:

$$
\begin{gathered}
E(t+1)=E(t)-D(t)+\eta_{s} G_{s}(t)+r(t) \eta_{c e} P_{c}(t), \\
X(t+1)=X(t)-L_{w}(t)+\eta_{c g} P_{c}(t)+\eta_{a g} P_{a}(t) .
\end{gathered}
$$

We then define the Lyapunov function $Q(t)=\frac{1}{2} E(t)^{2}+$ $\frac{1}{2} X(t)^{2}$. The conditional one-slot Lyapunov drift is:

$$
\Delta(t)=\mathbb{E}\{Q(t+1)-Q(t) \mid E(t), X(t)\} .
$$

Here, the battery queue and the water tank queue are of equal weight. Our algorithm can be extended if we assign different weights to them.

According to (25) and (26), by squaring both sides, we have:

$$
\begin{aligned}
\Delta(t) \leq & 0.5 \max \left[\left(\eta_{s} G_{s, \max }+\eta_{c e} P_{c, \max }\right)^{2}, D_{\max }^{2}\right] \\
& -E(t)\left[D(t)-\eta_{s} G_{s}(t)-r(t) \eta_{c e} P_{c}(t)\right. \\
& +0.5 \max \left[\left(\eta_{c g} P_{c, \max }+\eta_{a g} P_{a, \max }\right)^{2}, L_{w, \max }^{2}\right] \\
& -X(t)\left[L_{w}(t)-\eta_{c g} P_{c}(t)-\eta_{a g} P_{a}(t)\right] \\
= & B-E(t)\left[D(t)-\eta_{s} G_{s}(t)-r(t) \eta_{c e} P_{c}(t)\right] \\
& -X(t)\left[L_{w}(t)-\eta_{c g} P_{c}(t)-\eta_{a g} P_{a}(t)\right],
\end{aligned}
$$

where $P_{c, \max }$ is the maximum amount of the natural gas that can be used by the CHP in each time slot, and $B$ is a constant and defined as

$$
\begin{aligned}
B & =0.5 \max \left[\left(\eta_{s} G_{s, \max }+\eta_{c e} P_{c, \text { max }}\right)^{2}, D_{\max }^{2}\right] \\
& +0.5 \max \left[\left(\eta_{c g} P_{c, \text { max }}+\eta_{a g} P_{a, \max }\right)^{2}, L_{w, \max }^{2}\right] .
\end{aligned}
$$

According to the stochastic optimization framework, in order to make the two queues $E(t)$ and $X(t)$ mean rate stable, we must minimize the drift $\Delta(t)$. In addition, our control objective is to minimize the average cost. So we use a constant $V$ to represent the tradeoff between these two objectives. Then the drift plus penalty function can be written as follows.

$$
\begin{aligned}
\Delta(t) & +V \mathbb{E}\{f(t)\} \\
\leq B & -E(t) \mathbb{E}\left\{D(t)-\eta_{s} G_{s}(t)-r(t) \eta_{c e} P_{c}(t) \mid E(t)\right\} \\
& -X(t) \mathbb{E}\left\{L_{w}(t)-\eta_{c g} P_{c}(t)-\eta_{a g} P_{a}(t) \mid X(t)\right\} \\
& +V \mathbb{E}\left\{C_{e}(t)\left\{G_{l}(t)+G_{s}(t)-(1-r(t)) \eta_{c o} P_{c}\right\}\right. \\
& \left.+C_{g}\left\{P_{c}+P_{a}\right\}\right\} .
\end{aligned}
$$


We then substitute $G_{l}(t)$ in (29) according to (16), and after some manipulation we can obtain:

$$
\begin{aligned}
\Delta(t) & +V \mathbb{E}\{f(t)\} \\
\leq B & +V \mathbb{E}\left\{C_{e}(t) L_{e}(t) \mid E(t)\right\}-\mathbb{E}\left\{L_{w}(t) X(t) \mid X(t)\right\} \\
& -\mathbb{E}\left\{D(t)\left[E(t)+V C_{e}(t)\right] \mid E(t)\right\} \\
& +\mathbb{E}\left\{G_{s}(t)\left[\eta_{s} E(t)+V C_{e}(t)\right] \mid E(t)\right\} \\
& +\mathbb{E}\left\{P _ { c } ( t ) \left[r(t) \eta_{c e} E(t)+\eta_{c g} X(t)\right.\right. \\
& \left.\left.-(1-r(t)) \eta_{c o} V C_{e}(t)+C_{g} V\right] \mid E(t), X(t)\right\} \\
& +\mathbb{E}\left\{P_{a}(t)\left[\eta_{a g} X(t)+V C_{g}\right] \mid X(t)\right\} .
\end{aligned}
$$

Based on the "min-drift" principle of the Lyapunov optimization approach, the main idea of the proposed algorithm is to minimize the right-hand side (RHS) of (30) over all the feasible control policies in each time slot. In other words, at the beginning of each time slot, we observe the system states $B(t), W(t), L_{e}(t), L_{w}(t), C_{e}(t)$, determine the value of $B+V \mathbb{E}\left\{C_{e}(t) L_{e}(t) \mid E(t)\right\}-\mathbb{E}\left\{L_{w}(t) X(t) \mid X(t)\right\}$, and then solve the following problem:

\section{Problem Three (P-III)}

$\min G_{s}(t) H_{s}(t)+P_{c}(t) H_{c}(r(t))+P_{a}(t) H_{a}(t)-D(t) H_{d}(t)$,

subject to

$$
\begin{gathered}
L_{e}(t)=G_{l}(t)+D(t), \\
0 \leq \eta_{s} G_{s}(t)+r(t) \eta_{c e} P_{c}(t) \leq C_{\text {char }}, \\
0 \leq r(t) \leq 1, P_{c}(t), G_{l}(t), G_{s}(t) \geq 0 . \\
0 \leq D(t) \leq D_{\max },
\end{gathered}
$$

where

$$
\begin{aligned}
& H_{s}(t)=\eta_{s} E(t)+V C_{e}(t), \quad H_{a}(t)=\eta_{a g} X(t)+V C_{g}, \\
& H_{d}(t)=E(t)+V C_{e}(t), \quad H_{c}(r(t))=H_{r}(t) r(t)+H_{b}(t), \\
& H_{r}(t)=\eta_{c e} E(t)+\eta_{c o} V C_{e}(t), \\
& H_{b}(t)=\eta_{c g} X(t)-\eta_{c o} V C_{e}(t)+C_{g} V .
\end{aligned}
$$

Note that (31) contains the product of $P_{c}(t)$ and functions of $r(t)$, so P-III is a non-convex optimization problem because its Hessian matrix is not always positive definite. When looking into the structure of P-III, we can find that $D(t)$ and $P_{a}(t)$ can be easily obtained according to the value of $H_{d}(t)$ and $H_{a}(t)$. If $H_{d}(t) \geq 0$, then $D(t)=\min \left\{D_{\max }, L_{e}(t)\right\}$; otherwise $D(t)=0$. If $H_{a}(t) \leq 0$, then $P_{a}(t)=P_{a, \max }$; otherwise $P_{a}(t)=0$. Therefore, we only have to solve the following subproblem:

$$
\min : \quad G_{s}(t) H_{s}(t)+P_{c}(t)\left[H_{r}(t) r(t)+H_{b}(t)\right],
$$

subject to: (33) and (34).

Suppose (33) is not active and $0<r(t)<1$. Since $H_{c}(r(t))$ is a linear function of $r(t)$, then we can always increase or decrease $r(t)$ to make (37) smaller. Therefore, either (33) is active or $r(t)$ equals 0 or 1 .
Suppose (33) is active. We can replace $G_{s}(t)$ using (33) in (37) and get:

$$
\begin{aligned}
& \min : \quad\left(\eta_{c o}-\frac{\eta_{c e}}{\eta_{s}}\right) V C_{e}(t) P_{c}(t) r(t) \\
& +\frac{C_{c h a r}}{\eta_{s}}\left[\eta_{s} E(t)+V C_{e}(t)\right]+P_{c}(t) H_{b}(t) .
\end{aligned}
$$

Obviously, since $C_{e}(t), P_{c}(t) \geq 0$, if $\eta_{c o} \geq \frac{\eta_{c e}}{\eta_{s}}$, then $r(t)=0$; otherwise $r(t)$ should be as large as possible. If $\eta_{c e} P_{c, \max } \leq C_{c h a r}$, then $r(t)$ can be 1 and we can use this fact to convert P-III to a linear optimization problem by substituting $r(t)=0$ and $r(t)=1$ into P-III, respectively, and choose the minimum value. Otherwise, $r(t)$ should be in the range of $\left[C_{c h a r} / \eta_{c e} P_{c, \text { max }}, 1\right]$, and since this range is not too large, we can use a search algorithm to obtain the optimal solution.

Next we need to prove that the solutions to P-III are also feasible to P-I. In other words, the solutions to P-III can meet constraints (14) and (15) for $\forall t \in T$.

Theorem 1: Suppose $\theta$ and $\varepsilon$ are defined in (39) and (40), respectively,

$$
\begin{gathered}
\theta=\frac{V C_{e, \max }}{\eta_{s}}+\min \left\{D_{\max }, L_{e, \max }\right\} \\
\varepsilon=\frac{V C_{g}}{\eta_{a g}}+L_{w, \max } .
\end{gathered}
$$

Then through minimizing P-III, we can have the following results:

$$
\begin{aligned}
0 \leq & B(t) \leq \theta+C_{c h a r}, \quad \forall t \in T, \\
0 \leq W(t) \leq & \max \left\{\varepsilon+\frac{\eta_{c e} \theta-V C_{g}}{\eta_{c g}}+\eta_{c g} P_{c, \max },\right. \\
& \varepsilon+\frac{\eta_{c o} V C_{e, \max }-V C_{g}}{\eta_{c g}}+\eta_{c g} P_{c, \max }, \\
& \left.\varepsilon+\eta_{c g} P_{c, \max }+\eta_{a g} P_{a, \max }\right\}, \quad \forall t \in T,
\end{aligned}
$$

given that the above relationships are satisfied at $t=0$.

Proof. First, we use induction to prove the upper bound of $B(t)$ and $W(t)$. Since it holds when $t=0$, we assume it also holds at time slot $t$.

1) Suppose $B(t) \leq \theta$. Since $0 \leq \eta_{s} G_{s}(t)+r(t) \eta_{c e} P_{c}(t) \leq$ $C_{\text {char }}$, we can have $B(t+1) \leq \theta+C_{\text {char }}$.

2) Suppose $B(t)>\theta$. According to (23), $E(t)>0$. So we have $H_{s}(t)>0, H_{d}(t)>0$. To minimize P-III, it must be $G_{s}(t)=0$ and $D(t)=\min \left\{D_{\max }, L_{e}(t)\right\}$. Besides, we can find that $H_{c}(r(t))$ is an increasing function with $r(t)$, so $P_{c}(t) H_{c}(r(t))$ reaches its minimum value when $r(t)=0$. From all the above we can see whenever $B(t)>\theta$, the battery will discharge and do not charge. Therefore $B(t+1) \leq B(t) \leq \theta+C_{\text {char }}$.

3) Suppose $W(t) \leq \varepsilon$, it is obvious that $W(t+1) \leq \varepsilon+$ $\eta_{c g} P_{c, \max }+\eta_{a g} P_{a, \max }$.

4) Suppose $W(t)>\varepsilon$, then $X(t)>0$ according to (24), and $H_{a}(t)>0$. To minimize P-III, $P_{a}(t)$ must be 0 . Next we consider the following two cases. For the first case, if $\eta_{c e} E(t)+\eta_{c o} V C_{e}(t)<0, H_{c}(r(t))$ reaches its 
minimum value $-\eta_{c e} \theta+\eta_{c g}[W(t)-\varepsilon]+V C_{g}$ when $r(t)=1$ and $B(t)=0$. Therefore, when $W(t)>$ $\varepsilon+\frac{\eta_{c e} \theta-V C_{g}}{\eta_{c g}}, H_{c}(r(t))>0$ and $P_{c}(t)$ is set to 0 to minimize P-III. In this case, $W(t+1) \leq W(t)$. On the other hand, $W(t+1) \leq W(t)+\eta_{c g} P_{c, \max } \leq$ $\varepsilon+\frac{\eta_{c e} \theta-V C_{g}}{\eta_{c g}}+\eta_{c g} P_{c, \max }$.

For the second case, if $\eta_{c e} E(t)+\eta_{c o} V C_{e}(t) \geq 0$, $H_{c}(r(t))$ reaches its minimum value $-\eta_{c o} V C_{e, \max }+$ $\eta_{c g}[W(t)-\varepsilon]+V C_{g}$ when $r(t)=0$. Therefore, when $W(t)>\varepsilon+\frac{\eta_{c o} V C_{e, \max }-V C_{g}}{\eta_{c g}}, H_{c}(r(t))>0$ and $P_{c}(t)$ is set to 0 to minimize P-III. In this case, $W(t+1) \leq W(t)$. On the other hand, $W(t+1) \leq W(t)+\eta_{c g} P_{c, \max } \leq$ $\varepsilon+\frac{\eta_{c o} V C_{e, \max }-V C_{g}}{\eta_{c g}}+\eta_{c g} P_{c, \max }$.

From the above analysis, we can find that both $B(t)$ and $W(t)$ are upper bounded. So we can determine the capacity of the battery $B_{\max }$ and the capacity of the water tank $W_{\max }$ according to these upper bounds. Note that both $B_{\max }$ and $W_{\max }$ are functions of the parameter $V$, so we can make a tradeoff between the energy buffer capacity and the average cost. On the other hand, with a given battery pack or water tank capacity, we can obtain the corresponding parameter $V$.

Second, we also use induction to prove the lower bound of the battery and the water tank.

5) Suppose $B(t) \geq \min \left\{D_{\max }, L_{e, \max }\right\}$, obviously $B(t+$ 1) $\geq 0$.

6) Suppose $0 \leq B(t)<\min \left\{D_{\max }, L_{e, \max }\right\}$, substitute (39), (23) and (24) in (36) we can have $H_{d}(t)<$ $0, H_{s}(t)<0$. In order to minimize P-III, we must have $D(t)=0$ and $G_{s}(t) \geq 0$. Since $P_{c}(t) \geq 0$, $B(t+1) \geq B(t) \geq 0$.

7) Suppose $W(t) \geq \bar{L}_{e, \max }$, obviously $W(t+1) \geq 0$.

8) Suppose $W(t)<L_{e, \max }$, substitute (39), (23) and (24) in (36) we can have $H_{a}(t)<0$. In order to minimize P-III, we must have $P_{a}(t)=P_{a, \max }$. According to (8), we have $L_{w}(t+1) \geq L_{w}(t) \geq 0$.

Since both the SOC of the battery pack and the water level in the water tank are bounded, the solution to P-III is also feasible to P-I.

Theorem 2: If $C_{e}(t), L_{e}(t), L_{w}(t)$ are independent over slots, then the expected cost using the proposed algorithm over time is within bound $B / V$ of the optimal cost. In other words

$$
\lim _{T \rightarrow \infty} \frac{1}{T} \sum_{t=0}^{T-1} \mathbb{E}\left\{f^{\prime}(t)\right\} \leq P_{1}^{*}+B / V
$$

where $f^{\prime}(t)$ represents the energy cost in one time slot using the proposed algorithm, and $P_{1}^{*}$ is the optimal solution to P-I.

To achieve $P_{1}^{*}$, we need to know the distributions of the stochastic variables $C_{e}(t), L_{e}(t)$, and $L_{w}(t)$, which are difficult to obtain. Therefore, the proposed algorithm can provide a low-complexity approach to achieve a performance deviated no more than $O(1 / V)$ from the optimal one.

Proof. Assume $\left(D^{*}(t), r^{*}(t), G_{l}^{*}(t), G_{s}^{*}(t), P_{c}^{*}(t), P_{a}^{*}(t)\right)$ is the optimal policy to achieve $P_{1}^{*}$. Since the proposed algorithm

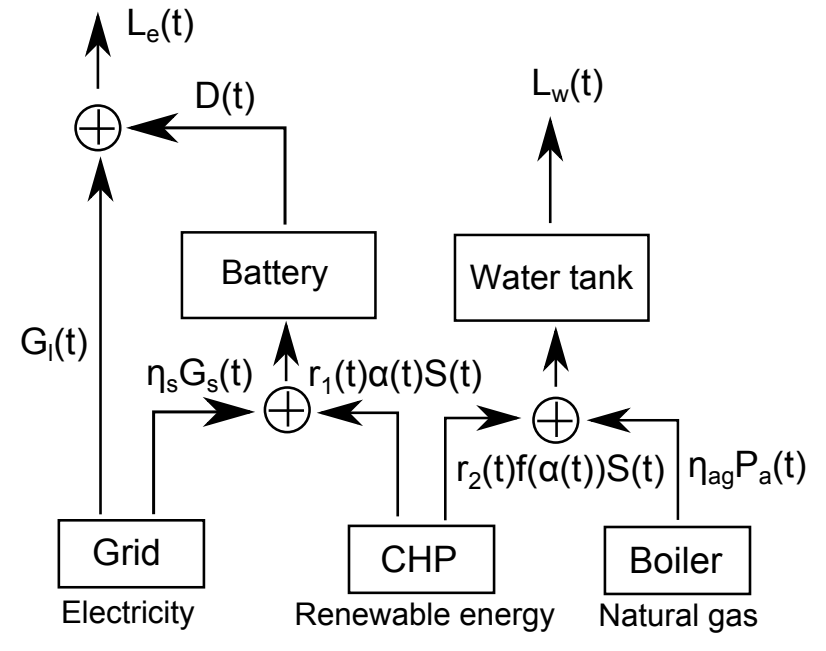

Fig. 2. CHP using renewable energy

is obtained by minimizing the RHS of (29), the value of the RHS of (29) should be no larger than that using the optimal policy. Then we have:

$$
\begin{aligned}
\Delta(t) & +V \mathbb{E}\left\{f^{\prime}(t)\right\} \\
\leq B & +V \mathbb{E}\left\{C_{e}(t) L_{e}(t) \mid E(t)\right\}-\mathbb{E}\left\{L_{w}(t) X(t) \mid X(t)\right\} \\
& -\mathbb{E}\left\{D^{*}(t)\left[E(t)+V C_{e}(t)\right] \mid E(t)\right\} \\
& +\mathbb{E}\left\{G_{s}^{*}(t)\left[\eta_{s} E(t)+V C_{e}(t)\right] \mid E(t)\right\} \\
& +\mathbb{E}\left\{P _ { c } ^ { * } ( t ) \left[r^{*}(t) \eta_{c e} E(t)+\eta_{c g} X(t)\right.\right. \\
& \left.\left.-\left(1-r^{*}(t)\right) \eta_{c o} V C_{e}(t)+C_{g} V\right] \mid E(t), X(t)\right\} \\
& +\mathbb{E}\left\{P_{a}^{*}(t)\left[\eta_{a g} X(t)+V C_{g}\right] \mid X(t)\right\} \\
\leq & B+V P_{1}^{*} .
\end{aligned}
$$

Taking the expectation on both sides, and summing over $t \in\{0,1,2, \cdots, T-1\}$, then we obtain

$$
\mathbb{E}\{Q(T)-Q(0)\}+\sum_{t=0}^{T-1} V \mathbb{E}\left\{f^{\prime}(t)\right\} \leq T B+T V P_{1}^{*} .
$$

Dividing both sides by $T V$, letting $T \rightarrow \infty$, and using the fact that both $Q(T)$ and $Q(0)$ are finite, we have:

$$
\lim _{T \rightarrow \infty} \frac{1}{T} \sum_{t=0}^{T-1} \mathbb{E}\left\{f^{\prime}(t)\right\} \leq P_{1}^{*}+\frac{B}{V} .
$$

\section{CHP USING RENEWABLE ENERGY}

In the previous section, we discussed the CHP system using the natural gas as the fuel. However, environmental concerns and the rising cost of fossil fuels make people to consider using renewable energy. Fortunately, some new CHP technologies can make use of certain renewable energy sources, such as Biomass and geothermal energy to generate both electricity and heat. In addition, as discussed in the CHPED problems, we can also adjust the ratio of generated electricity and heat in each time slot to optimize the energy cost. For example, we can use more geothermal energy to generate electricity and less to boil water if the electricity price is high, and vice versa. In 
this section, we discuss how to adjust the CHP generation ratio to minimize the average energy cost without the knowledge of the distribution of renewable energy generation, electricity and heat demands by using the Lyapunov optimization technique.

The system architecture is shown in Fig. 2. The electricity generation ratio from renewable energy is $\alpha(t)$, which ranges from $\alpha_{\min }$ to $\alpha_{\max }$, and the hot water generation ratio is a function of $\alpha(t)$, i.e., $f(\alpha(t))$, where $f$ is a decreasing function. $r_{1}(t)$ and $r_{2}(t)$, ranging from 0 to 1 , are used to avoid battery and water tank overflow. Since the renewable energy in a household is typically very limited, we assume $S_{\max } \alpha_{\max } \leq C_{\text {char }}$, where $S_{\max }$ is the maximum renewable energy available in one time slot. We will discuss the situation without this constraint later. We use a similar battery and water tank model as those in Section III, and the optimization problem can be formulated as follows:

\section{Problem Four (P-IV)}

$$
\min : \quad P_{4}=\frac{1}{T} \sum_{t=0}^{T-1} \mathbb{E}\left\{C_{e}(t)\left[G_{s}(t)+G_{l}(t)\right]+C_{g} P_{a}(t)\right\}
$$

subject to

$$
\begin{gathered}
B(t+1)=B(t)+\eta_{s} G_{s}(t)+r_{1}(t) S(t) \alpha(t)-D(t), \\
W(t+1)=W(t)+r_{2}(t) S(t) f(\alpha(t))+\eta_{a g} P_{a}(t)-L_{w}(t), \\
0 \leq B(t) \leq B_{\max }, \\
0 \leq W(t) \leq W_{\max }, \\
L_{e}(t)=G_{l}(t)+D(t), \\
0 \leq \eta_{s} G_{s}(t)+r_{1}(t) S(t) \alpha(t) \leq C_{c h a r}, \\
S_{\max } \alpha_{\max } \leq C_{c h a r}, \\
0 \leq G_{l}(t)+G_{s}(t) \leq P_{e, \max }, \\
0 \leq r_{1}(t), r_{2}(t) \leq 1, G_{l}(t), G_{s}(t) \geq 0, \\
0 \leq D(t) \leq D_{\max }, \\
\alpha_{\min } \leq \alpha \leq \alpha_{\max } .
\end{gathered}
$$

After we relax P-IV, the drift plus penalty function after manipulation can be written as follows:

$$
\begin{aligned}
\Delta(t) & +V \mathbb{E}\left\{C_{e}(t)\left[G_{s}(t)+G_{l}(t)\right]+C_{g} P_{a}(t)\right\} \\
\leq B^{\prime} & +V \mathbb{E}\left\{C_{e}(t) L_{e}(t) \mid E(t)\right\}-\mathbb{E}\left\{L_{w}(t) X(t) \mid X(t)\right\} \\
& -\mathbb{E}\left\{D(t)\left[E(t)+V C_{e}(t)\right] \mid E(t)\right\} \\
& +\mathbb{E}\left\{G_{s}(t)\left[\eta_{s} E(t)+V C_{e}(t)\right] \mid E(t)\right\} \\
& +\mathbb{E}\left\{\alpha(t)\left[E(t) r_{1}(t) S(t)\right] \mid E(t)\right\} \\
& +\mathbb{E}\left\{f(\alpha(t))\left[X(t) r_{2}(t) S(t) \mid X(t)\right]\right\} \\
& +\mathbb{E}\left\{P_{a}(t)\left[\eta_{a g} X(t)+V C_{g}\right] \mid X(t)\right\},
\end{aligned}
$$

where $B^{\prime}$ is a constant and defined as

$$
\begin{aligned}
B^{\prime}= & \frac{1}{2} \max \left[\left(\eta_{s} G_{s, \max }+\alpha_{\max } S_{\max }\right)^{2}, D_{\max }^{2}\right] \\
& +\frac{1}{2} \max \left[\left(\eta_{c g} P_{c, \max }+f\left(\alpha_{\min }\right) S_{\max }\right)^{2}, L_{w, \max }^{2}\right] .
\end{aligned}
$$

Our algorithm is to minimize the RHS of (59), i.e., to solve $\mathrm{P}-\mathrm{V}$.

\section{Problem V (P-V)}

$$
\begin{aligned}
\min & G_{s}(t) H_{s}(t)+\alpha(t) H_{h}\left(r_{1}(t)\right)+f(\alpha(t)) H_{f}\left(r_{2}(t)\right) \\
+ & P_{a}(t) H_{a}(t)-D(t) H_{d}(t),
\end{aligned}
$$

subject to (52) (53) (54) (55) (56) (57) (58),

where $H_{h}\left(r_{1}(t)\right)=E(t) r_{1}(t) S(t)$ and $H_{f}\left(r_{2}(t)\right)=$ $X(t) r_{2}(t) S(t)$.

Notice that $\mathrm{P}-\mathrm{V}$ is also a non-convex optimization problem. However, $r_{1}(t)$ and $r_{2}(t)$ must be 0 or 1 . To prove it, let's consider the following situations:

1) Suppose $E(t) \geq 0$, then both $G_{s}(t)$ and $r_{1}(t)$ should be 0 to minimize $\mathrm{P}-\mathrm{V}$ and avoid battery overflow.

2) Suppose $E(t)<0$ but $H_{s}(t) \geq 0$. We must have $G_{s}(t)=0$ and $r_{1}(t)=1$ to minimize $\mathrm{P}-\mathrm{V}$, and constraint (53) will not be violated due to (54).

3) Suppose $H_{s}(t)<0$, we have $E(t)<0$. Then constraint (53) is active or $r_{1}(t)=1$, otherwise we can increase $r_{1}(t)$ to further minimize P-V. Substituting $G_{s}(t)$ with $\left(C_{\text {char }}-r_{1}(t) S(t) \alpha(t)\right) / \eta_{s}$, and expanding $H_{s}(t)$ in (61), we have:

$$
\begin{aligned}
& \min : \quad-V C_{e}(t) / \eta_{s} S(t) \alpha(t) r_{1}(t) \\
& \quad+f(\alpha(t)) H_{f}\left(r_{2}(t)\right) P_{a}(t) H_{a}(t)-D(t) H_{d}(t),
\end{aligned}
$$

Since (54) guarantees that (53) will not be violated when $r_{1}(t)=1$, we have $r_{1}(t)=1$ to minimize (62).

4) Suppose $X(t) \geq 0$. Since $S(t) \geq 0$ and $0 \leq r_{2}(t) \leq 1$, we have $H_{f}(t) \geq 0$. Because $f(\alpha(t)) \geq 0, r_{2}(t)$ must be 0 to minimize $\mathrm{P}-\mathrm{V}$.

5) Suppose $X(t)<0$. Since $S(t) \geq 0$ and $0 \leq r_{1}(t) \leq 1$, we have $H_{f}(t)<0$. Because $f(\alpha(t)) \geq 0, r_{2}(t)$ must be 1 to minimize $\mathrm{P}-\mathrm{V}$.

By determining the values of $r_{1}(t)$ and $r_{2}(t)$ based on $E(t)$ and $X(t)$, we can convert P-V to a linear optimization problem with five variables which can be easily solved in real time. Note that constraint (54) is critical to make this problem convex. Without this constraint, we have to search $r_{1}(t)$ in the range of $\left[C_{\text {char }} / S(t) / \alpha_{\max }, 1\right]$ to obtain the optimal solution.

Theorem 3: Suppose $\theta$ and $\varepsilon$ are defined in (39) and (40), respectively. Then through minimizing $\mathrm{P}-\mathrm{V}$, we can have the following results:

$$
\begin{gathered}
0 \leq B(t) \leq \theta+C_{\text {char }}, \quad \forall t \in T, \\
0 \leq W(t) \leq \varepsilon+\eta_{a g} P_{a, \max }+S_{\max } f\left(\alpha_{\text {min }}\right), \quad \forall t \in T .
\end{gathered}
$$

given that the above relationships are satisfied at $t=0$, and

$$
\frac{1}{T} \sum_{t=0}^{T-1} \mathbb{E}\left\{f^{\prime \prime}(t)\right\} \leq P_{4}^{*}+B^{\prime} / V
$$

where $f^{\prime \prime}(t)$ represents the energy cost in one time slot using the proposed algorithm to minimize $\mathrm{P}-\mathrm{V}$, and $P_{4}^{*}$ is the optimal solution to P-IV. The proof of Theorem 3 is similar to that of Theorems 1 and 2 and thus is omitted due to space limitation. 


\section{Performance Evaluation}

We evaluate the performance of the proposed algorithms using real data. We consider a small hotel with 20 rooms equipped with a battery pack and a water tank. Their capacity are calculated based on the results in Theorem 1. In addition, we assume $\eta_{c o} \eta_{s}>=\eta_{c e}$ and $S_{\max } \alpha_{\max } \leq C_{c h a r}$, so both P-III and P-IV can be solved by convex optimization. If these conditions are not met, we can simply search within a small region to obtain the optimal result.

\section{A. Simulation Setup}

The real-time electricity price data we used in this simulation are obtained from [23]. The electricity sell-back price is assumed to be the same as the purchase price. The natural gas price is assumed to be a constant, $\$ 5.5 / M M B t u$, which is obtained from [1]. Meanwhile we used the wind-power generation data from [24] which has a time resolution of 15 minutes as the renewable energy source for the CHP system. The wind power is scaled down so that the maximum wind power generation equals $12 \mathrm{kWh}$ per hour. The time slot duration is set to be 15 minutes too. We assume that the original water temperature is 20 Celsius, so it need $111.11 B t u$ to heat one liter of water to 70 Celsius. The charging efficiency $\eta_{s}$ is set to 0.95 , and the boiler efficiency $\eta_{a g}$ is set to 0.8 . The default efficiency of the CHP is assumed to be $75 \%$ with $30 \%$ to generate electricity and $45 \%$ to generate heat. Since $1 k W h=3.41 k B t u$, we can have $\eta_{c o}=0.088 k W h / k B t u$ and $\eta_{c e}=0.0836 k W h / k B t u$. For the CHP with a variable generation ratio, we assume the total CHP efficiency is still $75 \%$, and the efficiency to generate electricity ranges from $20 \%$ to $40 \%$. Since the average power consumption is about $0.8 k W h$ per hour per user [26], we assume the electricity demand in one hour is uniformly distributed between 0 and $L_{e, \max }=32 \mathrm{kWh}$, while the hot water demand is also assumed to have a uniform distribution between 0 and $L_{w, \max }=200 L / h$. We fix the other parameters as follows (per hour): $D_{\max }=30 \mathrm{kWh}, C_{\text {char }}=20 \mathrm{kWh}$, $G_{l, \max }=G_{s, \max }=32 \mathrm{kWh}, P_{c, \text { max }}=0.05 \mathrm{MMBtu}$, and $P_{a, \max }=0.01 M M B t u$.

\section{B. Benchmark Algorithm}

We compare the performance of our algorithms with the situation without energy buffer, which is similar to the CHPED problem [2]. However, since the electricity and gas price model in the CHPED problem is different from ours, we did some changes to make them comparable. In each time slot, the controller chooses the control actions by solving the following optimization problem.

\section{Benchmark Algorithm I (B-I)}

$$
\min C_{e}(t) G_{l}(t)+C_{g}\left[P_{c}(t)+P_{a}(t)\right],
$$

subject to

$$
\begin{gathered}
G_{l}(t)+\eta_{c e} P_{c}(t) \geq L_{e}(t), \\
\eta_{c g} P_{c}(t)+\eta_{a g} P_{a}(t) \geq L_{w}(t), \\
G_{l}(t), P_{c}(t), P_{a}(t) \geq 0 .
\end{gathered}
$$

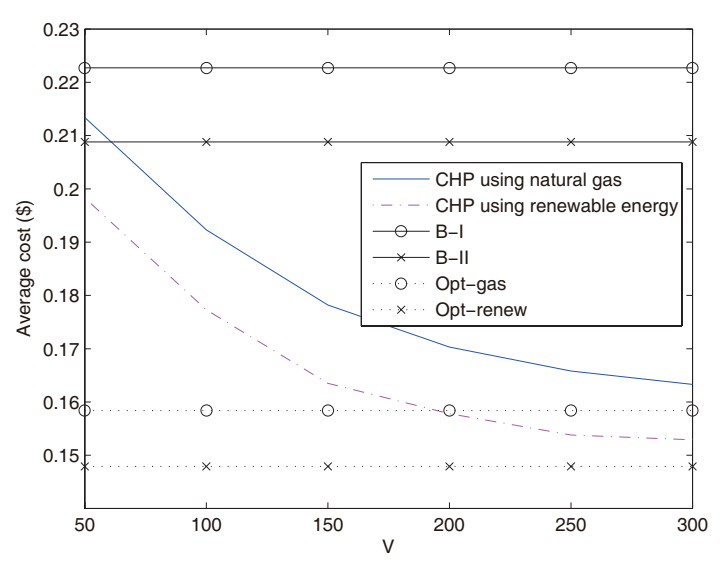

Fig. 3. Average cost with different V.

The objective of the optimization problem is to minimize the total cost in each time slot. Constraints (67) and (68) mean the electricity and heat generation in each time slot should be no less than the electricity and heat demand.

For the CHP using renewable energy, we have another corresponding benchmark algorithm.

\section{Benchmark Algorithm II (B-II)}

$$
\min C_{e}(t) G_{l}(t)+C_{g} P_{a}(t),
$$

subject to

$$
\begin{gathered}
G_{l}(t)+\alpha(t) S(t) \geq L_{e}(t), \\
f(\alpha(t)) S(t)+\eta_{a g} P_{a}(t) \geq L_{w}(t), \\
\alpha_{\min } \leq \alpha \leq \alpha_{\max }, \\
G_{l}(t), P_{a}(t) \geq 0 .
\end{gathered}
$$

The objective and constraints of B-II are similar as those of B-I, with the difference that we have different control actions and include renewable energy into the problem formulation.

\section{Results and Analysis}

Fig. 3 shows the average cost in one time slot for different parameter $V$. Due to the inherent exponential convergence property [25], the average cost decreases exponentially. The average cost of Benchmark Algorithm I and II in one time slot are $\$ 0.2227$ and $\$ 0.2088$, respectively. We can see the CHP system using natural gas can save up to $26.54 \%$ while the CHP system using renewable energy can save up to $28.63 \%$. In other words, with the help of energy buffers, the saving of using either CHP system can reach approximately $\$ 2,000$ annually. The saving mainly dues to the following reasons. First, with an energy buffer, the controller can use the electricity stored in the battery and make the CHP generate more electricity or even sell to the grid to make a profit when the electricity price is high and purchase electricity to charge the battery when the electricity price is low. Second, if the renewable energy source can generate more electricity and heat than required, we can store them in the energy buffer for future use. 


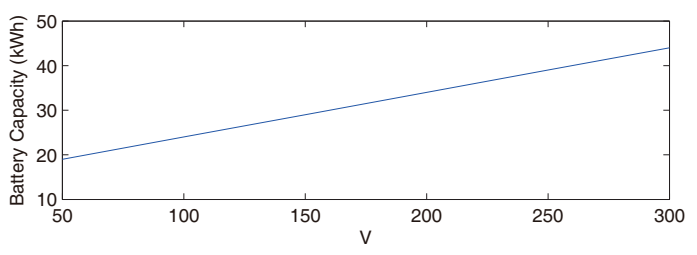

Fig. 4. The relationship between battery capacity and V

In Fig. 3, the optimal average cost in one time slot for the CHP using natural gas (Opt-gas) and renewable energy (Optrenew) are about $\$ 0.1584$ and $\$ 0.1479$, respectively. To obtain these results, we assume that the battery pack and water tank capacities are infinite and we can charge/discharge as much energy as possible to/from the battery when the electricity price is low/high. However, in practice, due to constraints (3) and (4), these values may not be achievable and are just provided here to show the possible bounds.

Fig. 4 shows the relationship between the required battery capacity pack and the parameter V. As was discussed in Theorem 1, the required battery capacity increases linearly with the increase of V. The capacity of the water tank has a similar relationship and is omitted due to the space limitation. With $V=200$, the battery pack and water tank capacity are $34 k W h$ and $419 L$, respectively. These values are quite reasonable for a small hotel with about 20 rooms.

\section{CONCLUSIONS}

In this paper, motivated by the queueing analysis and buffer management solutions in data communication systems, we have proposed an approach to minimize the average energy cost for two different types of CHP systems. Our system model includes renewable energy, real-time price, stochastic energy demand and energy buffers with finite capacity. Since the battery queue and the water tank queue are dependent, we need to solve a non-convex optimization problem to obtain the optimal control actions. By using the Lyapunov optimization techniques, our schemes can achieve a near-optimal performance, which will deviate no more than $O(1 / V)$ from the optimal solution.

In this paper, we assign equal weights to the battery and water tank queue which are dependent. In the future, we may adjust their weights to achieve a better performance according to the estimated distribution of the stochastic variables. In addition, to be applied in real scenarios, the capital expense of CHP systems, the operational expense associated with charging/discharging batteries, and the robustness of our algorithms also need further investigation.

\section{ACKNOWLEDGMENT}

This work is supported in part by NSERC, CFI and BCKDF.

\section{REFERENCES}

[1] ICF International Inc, "CHP Policy Analysis and 2011-2030 Market Assessment," Feb. 2012.
[2] F. Rooijers and R. Amerongen, "Static economic dispatch for cogeneration systems," IEEE Transactions on Power Systems, vol. 9, no. 3, pp. 1392-1398, 1994.

[3] G. Tao, M. Henwood, M. van Ooijen, "An algorithm for combined heat and power economic dispatch," IEEE Transactions on Power Systems, vol. 11, 1996.

[4] H. Liang, B. Choi, W. Zhuang, and X. Shen, "Optimizing the energy delivery via V2G systems based on stochastic inventory theory," IEEE Transactions on Smart Grid, vol. 4, no. 4, pp. 2230-2243, 2013.

[5] K. Zhou and L. Cai, "Randomized PHEV charging under distribution grid constraints," IEEE Transactions on Smart Grid, 2014.

[6] Z. Fadlullah, M. Fouda, N. Kato, A. Takeuchi, N. Iwasaki, and Y. Nozaki, "Towards intelligent machine-to-machine communications in Smart Grid," IEEE Communications Magazine, vol. 49, no. 4, pp. 60-65, 2011.

[7] L. Zheng, N. Lu, and L. Cai, "Reliable wireless communication networks for demand response control," IEEE Transactions on Smart Grid, vol. 4, no. 1, pp 133-140, 2013.

[8] A. Hawkes, M. Leach, "Cost-effective operating strategy for residential micro-combined heat and power," ELSEVIER Energy, vol. 32, pp. 711723, May 2007.

[9] K. Nanaeda, F. Mueller, J. Brouwer, and S. Samuelsen, "Dynamic modeling and evaluation of solid oxide fuel cell combined heat and power system operating strategies," Journal of Power Sources, vol. 195, no. 10, pp. 3176-3185, May 2010.

[10] M. Dentice, M. Sasso, S. Sibilio, L. Vanoli, "Micro-combined heat and power in residential and light commercial applications," Applied Thermal Engineering, vol. 23, pp. 1247-1259, July 2003.

[11] A. Lokurlu, T. Grube, B. Hhlein, D. Stolten, "Fuel cells for mobile and stationary applications cost analysis for combined heat and power stations on the basis of fuel cells," International Journal of Hydrogen Energy, vol. 28, pp. 703-711, July 2003.

[12] P. Subbaraj, R. Rengaraj, and S. Salivahanan, "Enhancement of combined heat and power economic dispatch using self adaptive real-coded genetic algorithm," Applied Energy, vol. 86, no. 6, pp. 915-921, 2009.

[13] T. Chang, M. Alizadeh, and S. Member, "Real-time power balancing via decentralized coordinated home energy scheduling," IEEE Transactions on Smart Grid, vol. 4, 2013.

[14] I. Koutsopoulos, "Optimal energy storage control policies for the Smart Power Grid,” SmartGridComm 2011, pp. 475-480, 2011.

[15] T. Hovgaard, K. Edlund, J. Jorgensen, "The potential of economic MPC for power management," 2010 49th IEEE Conference on Decision and Control, pp. 15-17, Dec. 2010.

[16] M. Neely, A. Tehrani, and A. Dimakis, "Efficient algorithms for renewable energy allocation to delay tolerant consumers," SmartGridComm 2010, pp. 549-554, Oct. 2010.

[17] R. Urgaonkar, B. Urgaonkar, M. Neely, and A. Sivasubramaniam, "Optimal power cost management using stored energy in data centers," ACM Performance Evaluation Review, vol. 39, no. 1, 2011.

[18] Y. Guo, S. Member, and M. Pan, "Optimal power management of residential customers in the Smart Grid," IEEE Transactions on Parallel and Distributed Systems, vol. 23, no. 9, 2012.

[19] L. Huang, J. Walrand, K. Ramchandran, and O. May, "Optimal demand response with energy storage management," [Online] Available: http: //arxiv.org/abs/1205.4297/

[20] Y. Huang, S. Mao, and R. Nelms, "Adaptive electricity scheduling in microgrids," Infocom 2013, pp. 1166-1174, 2013.

[21] M. Neely. Stochastic Network Optimization with Application to Communication and Queueing Systems. Morgan \& Claypool, 2010.

[22] L. Georgiadis, M. Neely, and L. Tassiulas. Resource allocation and cross-layer control in wireless networks. Now Publishers Inc., 2006, vol. 1.

[23] "The Electric Reliability Council of Texas," [Online] Available: http: //www.ercot.com/

[24] "Wind energy generation data sets," [Online] Available: http://www. eirgrid.com/operations/systemperformancedata/windgeneration/

[25] J. Slotine and W. Li. Applied Nonlinear Control. Prentice Hall, 1991.

[26] California Energy Commission, "Residential Appliance Saturation Study," [Online] Available: http://www.energy.ca.gov/appliances/rass/

[27] D. P. Bertsekas. Dynamic Programming and Optimal Control, 2nd ed. Athena Scientific, 2000. 